\title{
Development and testing of a visualization application software, implemented with wireless control system in smart home care
}

\author{
Jan Vanus, Pavel Kucera, Radek Martinek and Jiri Koziorek
}

* Correspondence: jan.vanus@vsb.cz VSB TU Ostrava, Department of Cybernetics and Biomedical Engineering, Technical University of Ostrava, 17. listopadu 15/2172, 708

33 Ostrava, Czech Republic

\begin{abstract}
This article describes the development of a visualization application software used to control operational and technical functions in the Smart Home system or Smart Home Care system via the wireless xComfort control system. Graphic visualization of a home electrical control system gives the user unprecedented comfort when controlling home systems. The user is able to obtain the information necessary to optimise the management of operational and technical functions in the building as well as information about energy consumption. Selected definitions of requirements for the visualization system, online access via the Internet, control via USB interface, and control requirements executed via mobile phone are the reasons why these technical elements were selected. This article describes their mutual relations, functions and connections within the system. At the end of this article we propose a method to test the reliability of the created software application as well as the wireless xComfort system under different conditions which stimulate different implementation methods applicable to a real building/ apartment unit. Measurement results can be used for the actual installation process and for optimal implementation of the active elements of the wireless system.
\end{abstract}

Keywords: Visualization; Wireless system; Smart home; Smart home care; Testing; Control

\section{Introduction}

Together with a growing standard of living in economically advanced countries, the human life is prolonged, which results in increased representation of elderly people in the society in Czech Republic (Figure 1). Their growth makes the society think about the specific needs of this age group [1]. With changes in life expectancy across the world, technologies enhancing well-being of individuals, specifically for older people, are subject to a new stream of research and development [2]. The nature of miniature wireless sensors and rapid developments in the wireless network technology have revolutionized home monitoring and surveillance systems. The new means and methods of collecting data efficiently and have led to novel applications for indoor wireless sensor networks [3]. Advances in computing, smart homes, and sensor technologies enable novel, longitudinal health monitoring applications in the home. Many home monitoring technologies have been proposed to detect health crises, support aging-in-place, and improve medical care [4]. To address the need for autonomous control of remote and distributed mobile

2014 Vanus et al.; licensee Springer. This is an Open Access article distributed under the terms of the Creative Commons Attribution License (http://creativecommons.org/licenses/by/4.0), which permits unrestricted use, distribution, and reproduction in any medium, provided the original work is properly credited. 


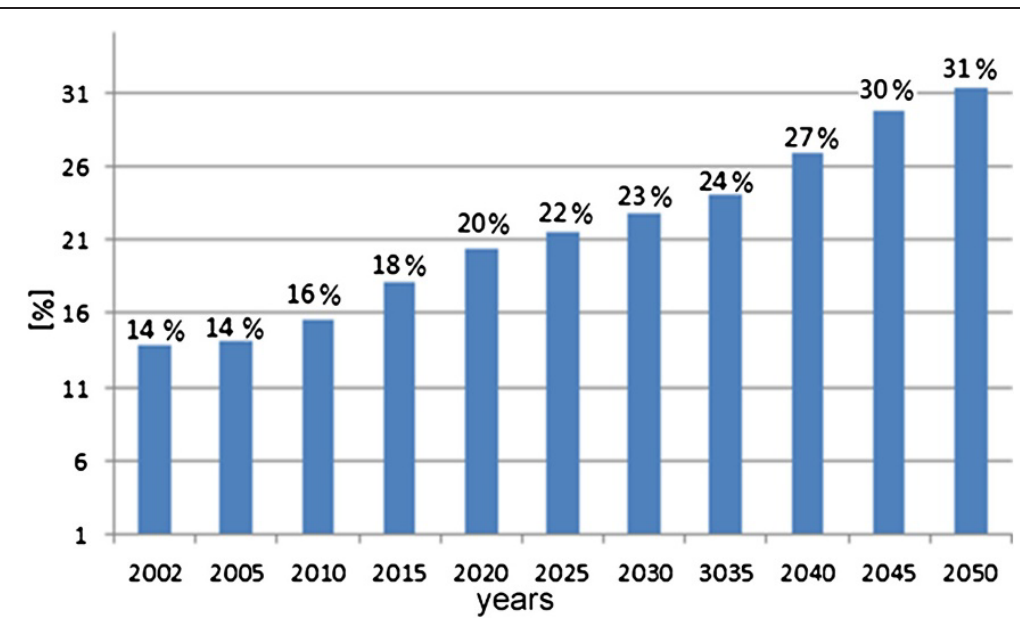

Figure 1 Population prognosis of Czech citizens older than 65 from 2002 until 2050 -the middle version (the Czech Statistical Office) [6].

systems, Machine-to-Machine (M2M) communications are rapidly gaining attention from both academia and industry. M2M communications have recently been deployed in smart grid, home networking, health care, and vehicular networking environments [5].

A considerable part of the behavior in smart environments relies on event-driven and rule specification. Rules are the mechanism most often used to enable user customization of the environment [7]. Some research workers propose socially acceptable physiological signal monitoring system consisting of a natural sensing interface and an intuitive information display [8]. Other studies in economic psychology have repeatedly revealed that energy consumption behavior in the household could be optimized through the provision of frequent and immediate feedback [9-11]. Another system is designed to make integration and interconnections of new metering devices and visualizations simple and constitutes a good basis for further research on awareness of energy usage in our (future) living environments [9]. Next was presented a fully distributed system and architecture based on a network of smart cameras to perform georeferenced tracking and activity recognition. Additionally an intuitive visualization was presented, where all information were integrated in one world model that is available ubiquitously [12]. A different paper proposes a new way of providing virtual smart home services using tangible mixed reality (MR), which provides more cost-effective and reliable visualization and simulation of the existing pervasive environment [13]. Other way of visualization is new HMI (HumanMachine Interface) system, which is based on statically positioned indicators for data output and a touch screen above them for data input. Indication elements consist of discrete light emitting diodes (LED), LED matrices, segment-type displays and small sized graphical visualization panels [14]. Some studies focuses on the application-layer simulation to propose a Visualization System of Context-aware Application Scenario Planning (VS-CaSP) for assisting non-technical developers and end-users in rapidly and easily designing the application scenario of smart buildings and in performing the acceptable and predictable simulation and evaluation [15]. The promising trend of visualization technology for textiles facilitates home consumers to approach and access both market information and application guidelines with easy means such as a remote controller, therefore, may help to promote the home textile consumptions significantly [16]. One of 
key components in the development of smart home technology is the detection and recognition of activities of daily life. Based on a self-adaptive neural network called Growing Self-Organizing Maps (GSOM), was presented a new computational approach to cluster analysis of human activities of daily living within smart home environment [17].

In connection with the dynamic progress of research and development of the above mentioned Tele Care, Health Care, Smart Home, Smart Home Care, Smart Grids, Smart Cities, Smart Metering technologies we would like to react to these trends by creating a platform for testing of new technologies. One of the segments of the built platform is the area of the support of elderly citizens living independently in their homes. There are efforts to use the above-mentioned current modern Smart Home Care technologies in terms of convenient controlling of home operational and technical functions by citizens over 65 years of age. Some companies in Czech market offer remote convenient controlling of Smart Home operational and technical functions using all available standard technologies such as mobile phones, tablets, iPods, iPads, iPhones and suchlike. However, in order to be connected to the supplied bus or wireless system, it is necessary to pay extra money for the license or the corresponding communication interface. The article focuses on the description of development and implementation of the visualization software of the application for controlling of home operational and technical functions within Smart Home Care using wireless xComfort system with subsequent testing in the environment of an apartment house.

\section{Describe of the created visualization appliaction SmartHomeApp}

In order to support the possibility of senior citizens living independently in their homes, we have created in our business a visualisation application called SmartHomeApp for intuitive, comfortable and remote controlling of home service systems within Smart Home Care (Figure 2).

In terms of the actual implementation of wiring, it is desirable to use technologies utilizing wireless technology (actuators and sensors) in houses with the already existing wiring. For control operational and technical functions in Smart Home and Smart Home Care, xComfort wireless technology is used. This technology uses $868.3 \mathrm{MHz}$ radio frequency for communication. Transmission takes up a maximum of $1 \%$ of the entire time. Data are being confirmed during radio frequency communication between the actuator and the sensor. Data transfer may be protected by a password. Increase of the reach of RF signal between individual components is possible thanks to automatic signal transfer call Routing .

In terms of communication between the database and the visualization and active elements, a software driver was designed, which makes this communication possible. The developed visualization software SmartHomeApp was designed with regard to web interface requirements, ability to control the software via a mobile phone, via voice control and also with regard to easy expendability, scalability and modularity. The system consists of three logical parts (Figure 3):

user interface,

controlling computer,

USB interface/active elements. 


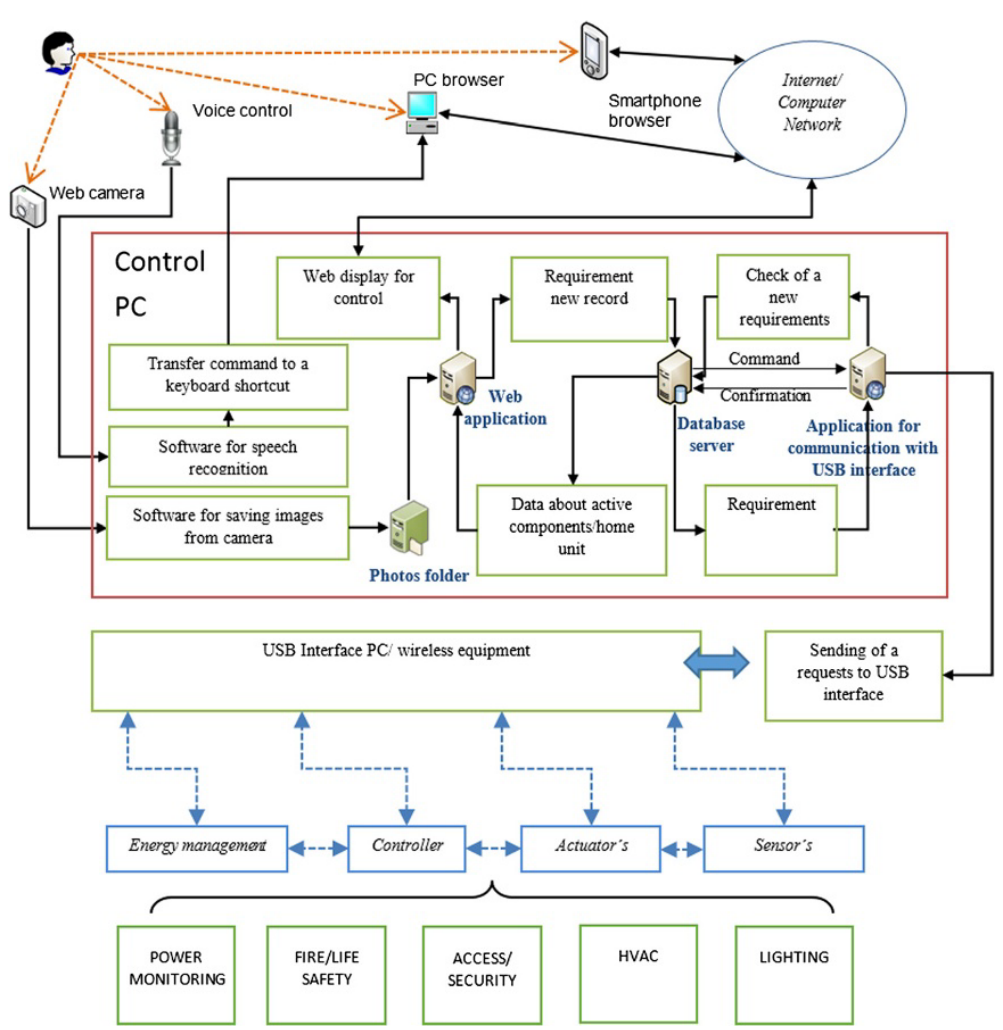

Figure 2 Block diagram of the designed visualization software SmartHomeApp for control, monitoring and visualization of operational and technical functions in Smart Home or Smart Home Care with xComfort wireless technology.

User interface acts as a layer between the user and the controlling computer. It displays system status information and provides inspection and control elements used to control active elements in the apartment.

The controlling computer secures interconnection between a device using USB interface and the user interface. It provides an environment for smooth operation of software elements used for active element control process. It accepts and registers whole system requests. It checks and sends changes, i.e. requests for system changes. It accepts change confirmations and forwards them to the system and to the user interface. It also stores information used later for optimization of system actuators installed in the housing unit. USB interface sends requests to active elements in the apartment and receives answer which is then forwarded to the control computer. By interconnecting these layers, it is possible to implement the created visualisation system for controlling of home operational and technical functions using wireless xComfort system. The process of model implementation of SmartHomeApp visualisation software:

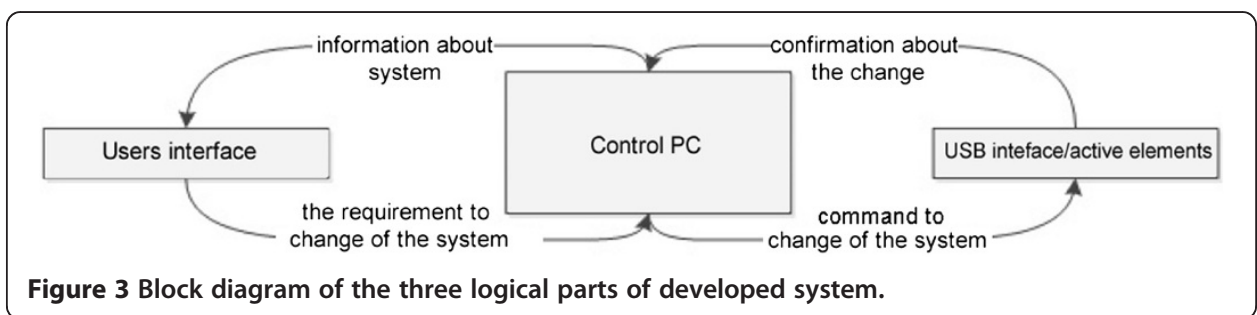


1. Creation of an apartment layout.

2. Specifications of used components.

3. Analysis of communication protocol and testing of packets.

4. Creation of visualisation application software (analysis, visualization implementation process).

5. Description of the use of other software applications.

6. Application testing.

\section{Creation of apartment layout}

The created visualisation of a model housing unit includes a hallway, bathroom, kitchen joined with the living room and a workroom joined with the sleeping room. The visualization is implemented as a clickable map (Figure 4). The floor layout of the apartment unit serves as the base for the clickable map. For the needs of the visualisation, the layout is divided into logical blocks according to rooms and the manner of their utilization. This division gives the user better view of rooms and simpler access to the desired active element.

\section{Specifications of used components}

Further it was necessary to specify individual components of xComfort wireless system with subsequent programming, the so-called setting of links between actuators using software tool EATON RF-System. The mutual cohesion of actuators and sensors may be monitored according to the lines on the computer screen (Figure 4).

\section{Analysis of communication protocol and testing of packets}

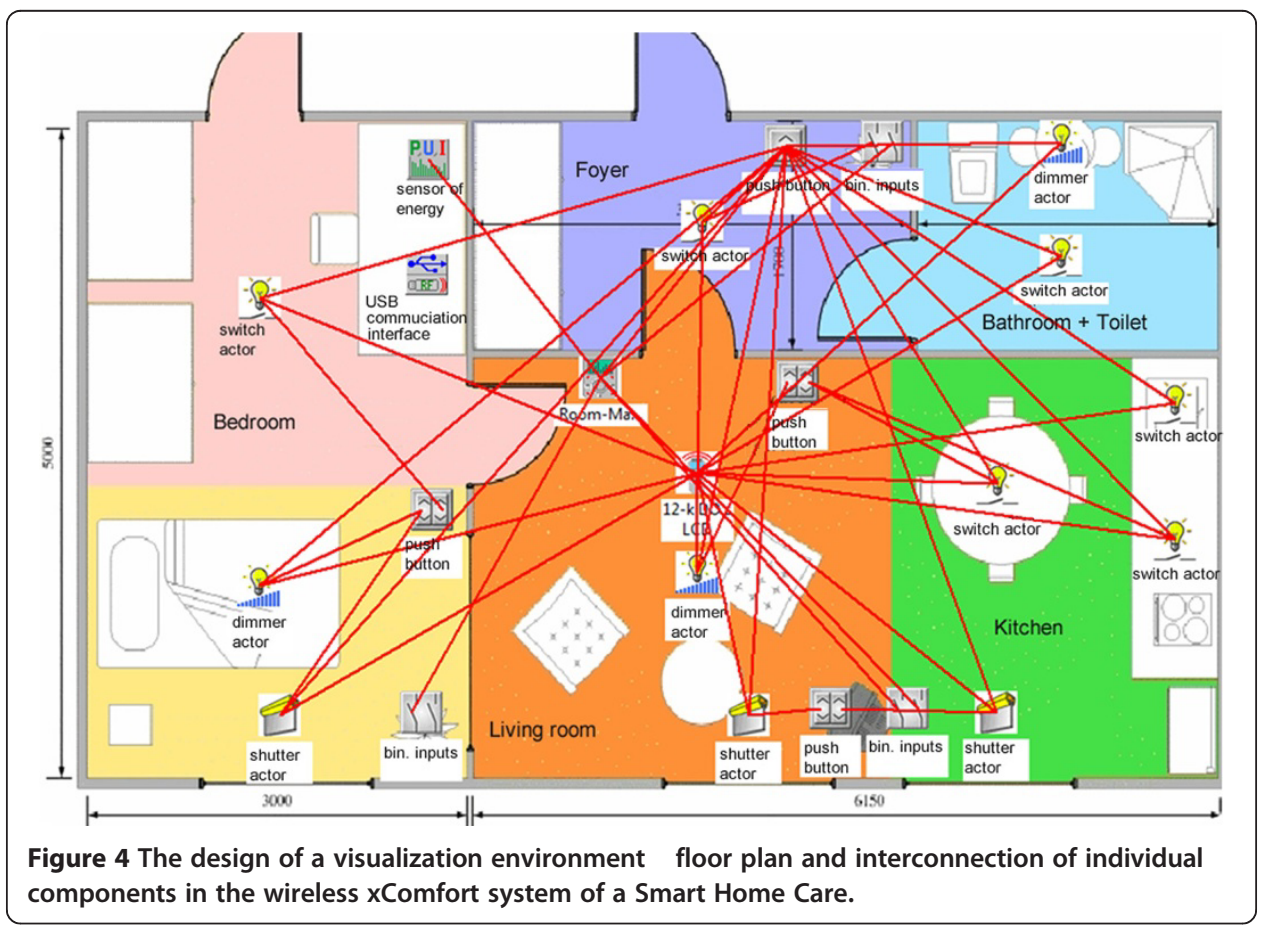


The communication protocol describes communication between individual components of xComfort wireless technology. The owner of this protocol is Eaton and its use and publishing is subject to contractual arrangement. The document describes in detail the communication in the Basic and the Comfort Mode. The following components were used for testing of the communication protocol:

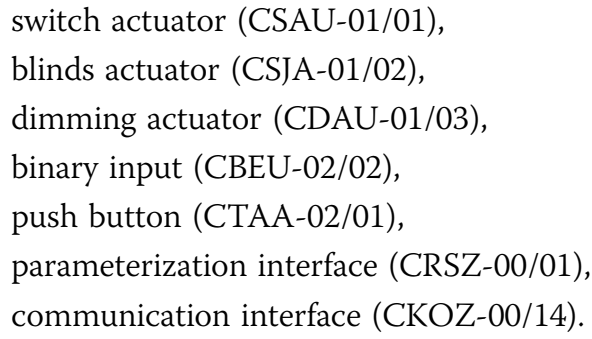

The shape of the packets sent to USB interface is defined in the communication protocol. The test was implemented on active connection elements of testing case using SimpleHIDWrite software. The following commands were used for controlling of the above mentioned actuators:

$$
\begin{aligned}
& \text { switch on, } \\
& \text { switch off, } \\
& \text { brighter } \\
& \text { darker, } \\
& \text { stop, } \\
& \text { fully close, } \\
& \text { fully open, } \\
& \text { close one step, } \\
& \text { open one step. }
\end{aligned}
$$

4. Description of the design of visualization and application software

It is a set of technical solutions covering various levels of controls, monitoring and visualization of statuses of controlled devices (xComfort wireless system actuators). One of the main parts of the system is an SQL server, where all system statuses and control instructions are saved. The control instructions are recorded. Each new record is recognized by the application and sent through control interface to the respective controlled elements. Then the controlled element sends information confirming whether the request was actually carried out. If the information specifying that the request was done is received, the status of the control device will change and the request is marked as completed. Here, the control unit represents visualization software, displays statuses of individual devices and options for their configurations. By using the database server it is possible to monitor the recorded instructions and processes, to evaluate results of individual instructions and thus find an optimal solution. The actual implementation of visualization was divided into multiple stages:

Analysis of SmartHomeApp web visualization, 


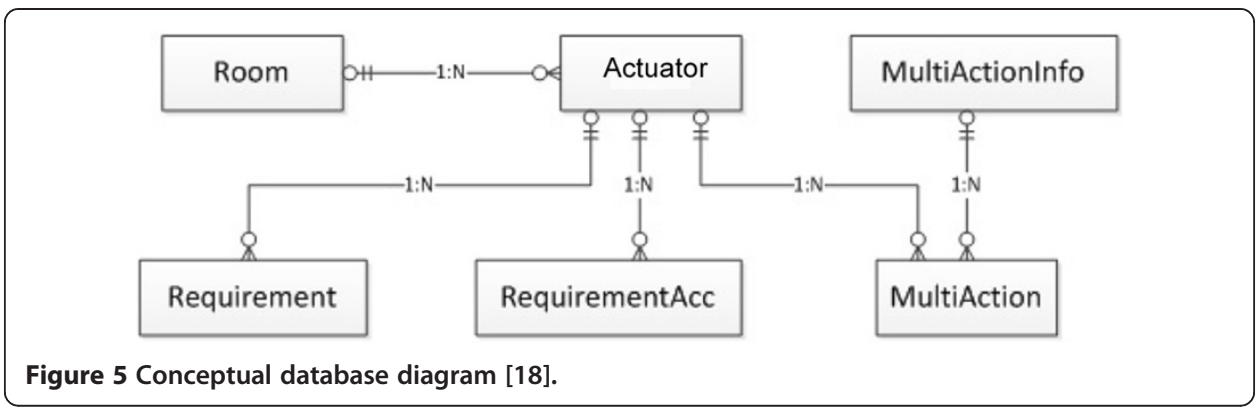

Data analysis,

USBinterfaceTransfer software analysis,

Visualization implementation process client,

Visualization implementation process server.

Analysis of SmartHomeApp web visualization

Web visualization SmartHomeApp is used by the user to control intelligent electrical system components. The key function is to control actuators through direct changes in the electrical system and collective/bulk modification of the electrical system [18].

\section{Data analysis}

Data analysis is described through a conceptual database diagram (Figure 5) and through a database scheme (Figure 6) with data dictionaries/terms used in Requirement, RequirementAcc, MultiActionInfo, MultiAction, Room and Actuator tables.

\section{USBinterfaceTransfer software analysis}

The USBinterfaceTransfer tool is used to send requests to USB interface. USB interface will send requests to active elements in the intelligent electrical system. The program repeatedly searches for incomplete/unexecuted records and then marks them as completed/done [18].

\section{Visualization implementation process client}

Selection of technical elements necessary for the respective visualization is directly dependent on our efforts to make actuator controlling accessible via web browser and through a computer network or the Internet (Figure 7).

This part contains descriptions and sample uses of technical elements, which were used to create visualization environment for the client/at the clients facility. The client represents a web browser used by the user to access the application. If voice control system is used, it refers to an add-on program, which controls the web browser. Information provided to the user of the relevant web browser is dynamically loaded from the database, in this situation from MS SQL.

Transfer of information between the control computer and client is done via HTTP protocol. Document is structured using HTML and SCC. JavaScript is used as a higher form of a comfortable control system [19]. 


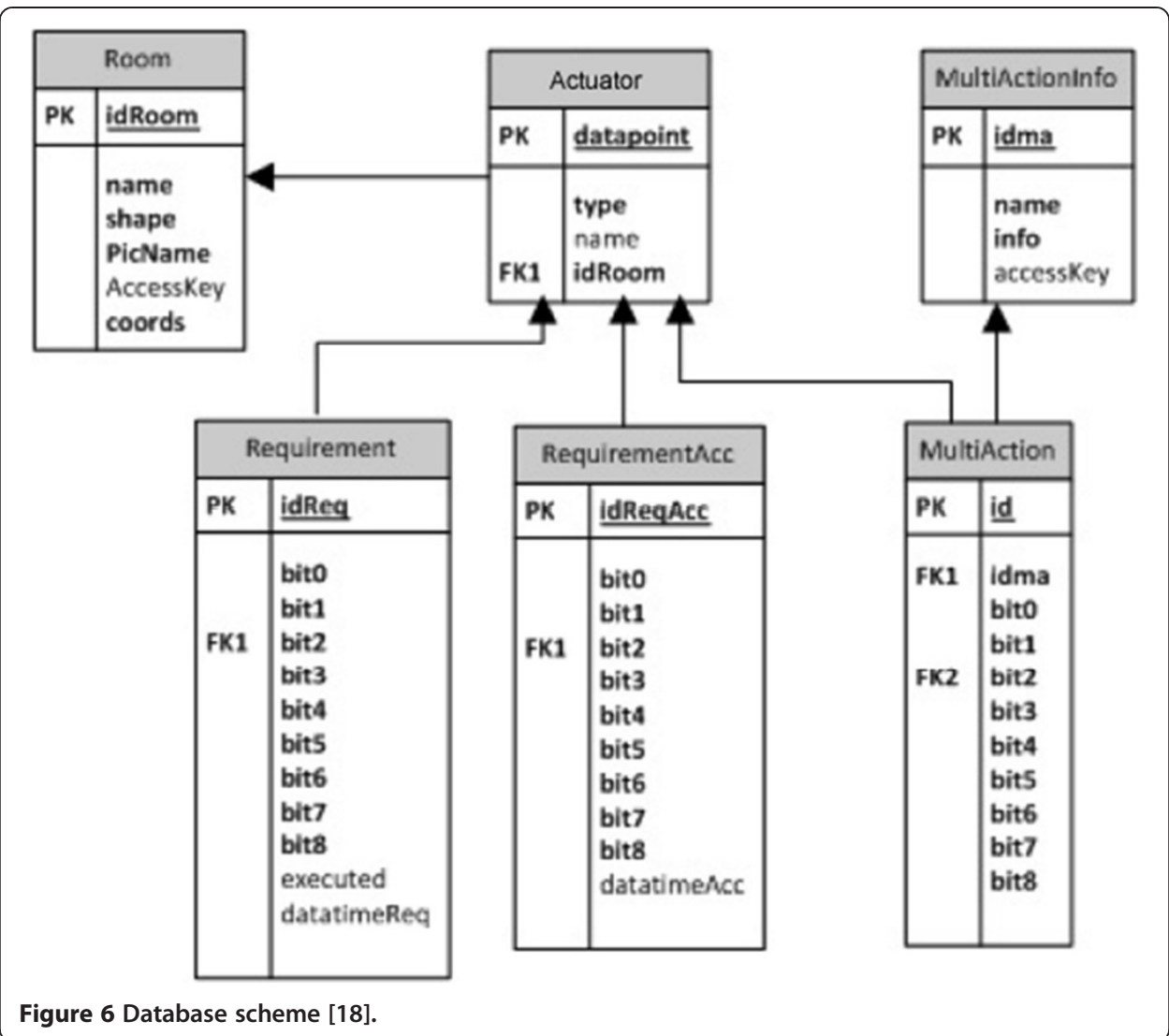

\section{Visualization implementation process server}

System elements in server are selected based on new trends, scalability and easy system expandability. The server side refers to all logical software products ensuring proper operation of applications used to control xComfort intelligent electrical installations. To achieve this, two programs are used: SmartHomeApp and USBinterfaceTransfer . The first program is created in ASP.NET environment, and the second one in .NET environment and both use C\# language [19].

\section{Software Tools Used in the Visualization Application}

Software tools used in the application are described below:

Web Server

A computer program responsible for resolving http clients requests. For a web server, a web browser is considered to be the client. It is an essential part of web applications operation. The web server sends its response as a HTML document; it can be created dynamically, using, for example, PHP or ASP technology.

Database Server

The following section describes SmartHomeApp database structure, process of its creation and usage. The entire database is a subject of an analysis; just most important schemes will be described here. 


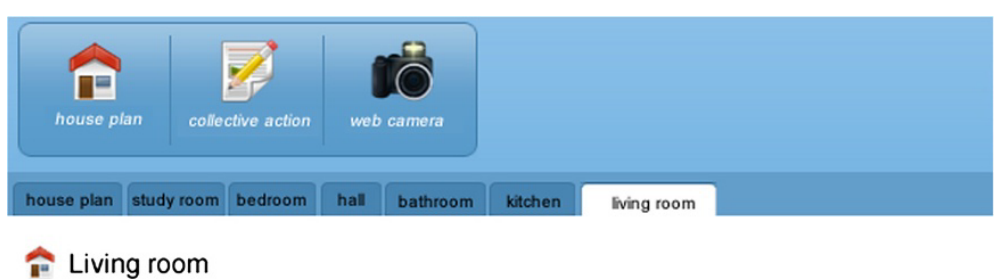

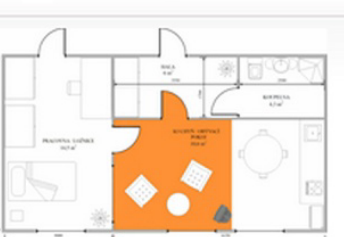

House Plan

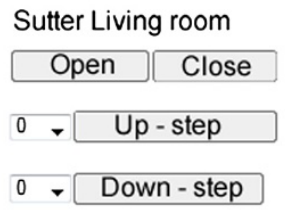

Dimming Living room

\begin{tabular}{|c|c|}
\hline Turn on & Turn off \\
\hline brighter & darker \\
\hline
\end{tabular}

Light Living room

Turn on Turn off

Figure 7 The design of a visualization environment control of operational and technical functions in Smart Home Care [19].

Database creation:

CREATE DATABASE Smart Home COLLATE Czech CI AS; This command created the Smart Home database, and Collate command to set a character set was established. Czech character set was set by COLLATE Czech CI AS command which is caseinsensitive $(\mathrm{CI})$ and diacritics-sensitive.

Room table creation

Contains information about the room, vital to run the SmartHomeApp .

CREATE TABLE [dbo].[Room](

[idRoom] [int] PRIMARY KEY NOT NULL, [name] [nvarchar](50) NULL, [shape] [nvarchar](50) NULL, [coords] [nvarchar](50) NULL, [PicName] [nvarchar](50) NULL, [accessKey] [nchar](1) NULL) idRoom -It serves as a unique room identifier in the database. Name -Room name to display in the system.

Shape -A basic shape to draw the room on the flat map. Coords -Flat size coordinates.

PicName -An image file, showing room position drawn in.

accesKey -A selected shortcut key, necessary for simple, trouble free voice control. 
PRIMARY KEY NOT NULL rule defines the column as a primary key, UNIQUE by default, but the UNIQUE clause can $t$ be present.

Actuator table creation

Contains information about all actuators in the intelligent home system, which have to be controlled.

CREATE TABLE [dbo]. [actuator]

CREATE TABLE [dbo].[actor](

[datapoint] [bit] PRIMARY KEY NOT NULL,

[type] [bit] NULL,

[name] [nvarchar](50) NULL,

[idRoom] [int] NULL)

Datapoint Unique

actuator identifier in the system.

Type -Actuator type, blind or other.

Name -Name, actuator label in the system. It shows during the control process.

idRoom -Room identifier.

Requirement table creation

Contains commands for active elements of the flat. It contains both commands executed by saving a value in the executed column and commands waiting to be executed.

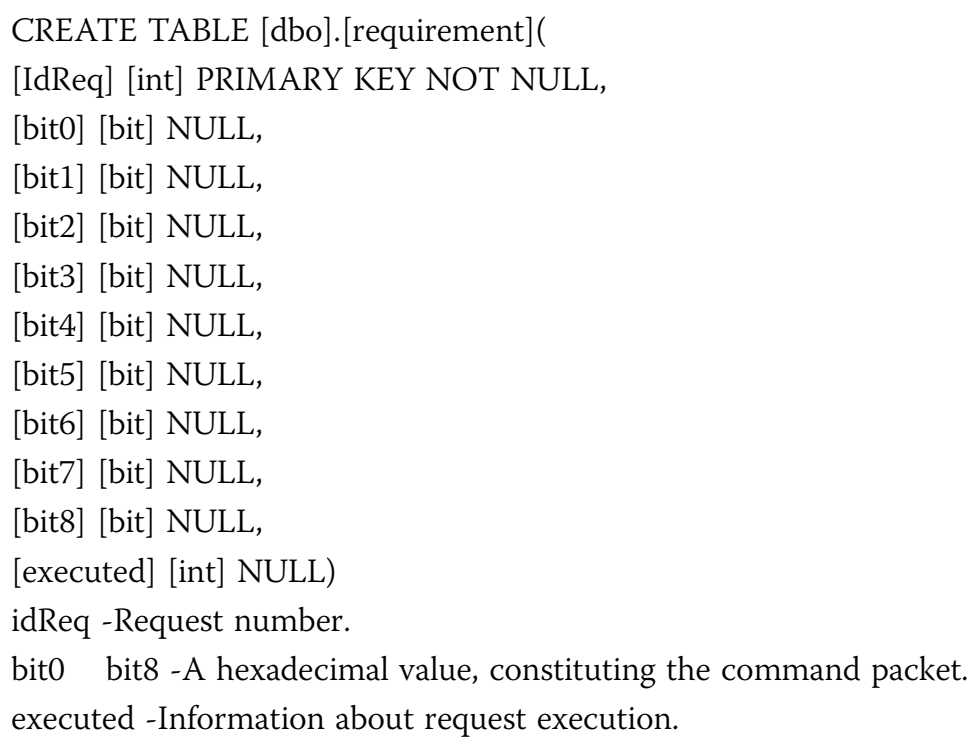

The above mentioned described the database core for active xComfort elements visualization.

By design, database engine usage doesn $t$ matter. However, it is important to keep information about rooms and their attributes, and this goal is accomplished by the Room table. It is important keep a record which actuator belongs to a room, its type for different control and a number, under which it is registered in the active elements system, and, finally, saving requests for system changes. Command records enable to use the 
commands later during analyses. The database schema described above is not very suitable to analyze system behavior but it provides us with a core to run the system. To analyze operation and technology tools of the flat it is necessary to extend the system.

Web Application

SmartHomeApp web application is visualization software of the proposed system. It is a web application built on ASP.NET technology. One of the core requests to run a web application is a web server. To run an application for .NET, a framework is necessary. SmartHomeApp requires Framework 3.5. The best tool to meet requirements of a web application based on ASP.NET architecture is IIS (Internet information services). It is a set of Microsoft applications suited for Internet, including a web server. Thanks to its integration into the operating system it can cooperate with applications built on .NET very well and it can run web applications merged with other infrastructures [19].

\section{USBinterfaceTransfer}

USBinterfaceTrancfer helps to ensure communication between the database and USB communication interface. It is loosely based on the Generic HID open source library from EATON. The program is based on the .NET Framework 2.0 platform and written in C\# language.

It is designed to keep detecting new records with new requests from the database. If a new request is found, the tool sends it to the USB communication interface.

MyVoice

The MyVoice software tool serves to recognize voice commands. To control SmartHomeApp visualisation software by voice, a shortcut key control method was selected. It is necessary to keep perfect records about using defined keys for shortcuts. To fully control the system by voice, shortcuts have to be set for every feature, from menu keys to the room list to each function.

Generally, the browser prefers a HTML document shortcut key, but for MyVoice the situation is different. For voice control of technical functions is necessary solve problem with additive noise in speech signal [20].

Webcam

A webcam continually saves photos and then the images are displayed in a web browser. This principle is used by many computer programs. The Camera 2004 tool is used in the project. When designing the Smart Home Care with assistance service, the Distributed Direct Sensing system can be used. With regard to the senior citizens needs (respecting the privacy), it is necessary to activate the camera in dependence on evaluation of a predefined unexpected event (water overflow, detection of smoke, etc.). The citizen can decide when the camera should be activated. It is clear that modern technologies have only a supporting function in a comprehensive approach to the senior citizens needs [21].

6. Testing the Reliability of Wireless System for Operational and Technical Functions Control in Smart Home

Signal quality measuring method between the actuators (switching, dimming, window blinds) and the USB interface was selected to test the reliability of operational and technical functions which are controlled wirelessly in a Smart House using the proposed visualization application software (Figure 8).

In predetermined distances was placed a computer with visualization software 


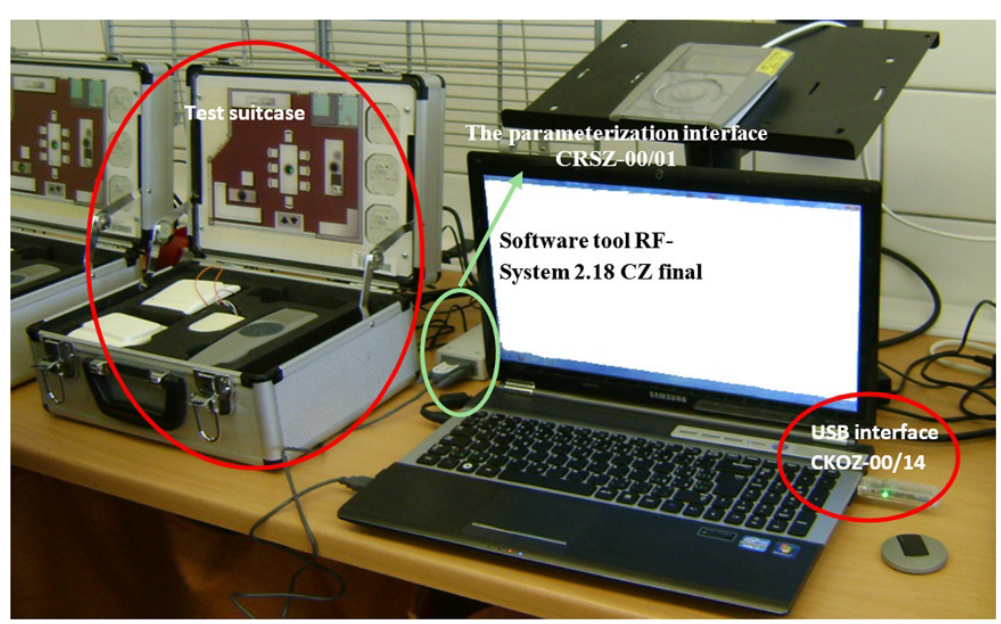

Figure 8 The laboratory workspace for design, programming, measurement, control and simulation of operational and technical functions in the Smart Home Care with using of the wireless xComfort system.

SmartHomeApp together with a connected USB interface CKOZ-00/14 and a testing briefcase containing a model of an apartment unit (Figure 9), (Figure 10), (Figure 11). Measurements were performed using parameterization interface CRSZ-00/01 and software tool Eaton RF-System 2.18 CZ Final. Then was measured of maximum communication distance between the actuator (switching, dimming, window blinds) and USB communication interface in all measured environments with using of the created visualization program SmartHomeApp .

\section{The distance /[m] measurement -environment without any obstacles}

An ideal status is if no solid obstacles stand in the way of the radio frequency signal travelling between the transmitter and receiver (Figure 9). Measurements showed huge differences in signal reception, mostly between window blinds and other actuators (Figure 12), (Table 1). Measurements also included testing of maximal signal reach for active switching element. Through additional testing was confirmed that USB interface using SmatHomeApp visualization operates reliably within a distance up to 150 meters.

During these measurements was established the optimal distance $20 \mathrm{~m}$ for switch actuator and dimming actuator and $10 \mathrm{~m}$ for shutter actuator (Table 1). The parameterization interface experiences serious problems trying to find active components in distances over 20 meters. This also limits the measurement of the signal quality.

\section{The distance /[m] measurement -environment with one brick wall}

Brick materials are the most common materials used to build houses and apartments. Brick wall testing represents a real life model and practical application (Figure 10). Signal loss due to a brick wall is directly connected to the thickness of the wall even though brick clay is a material with good properties when compared with other materials such as reinforced concrete structures or metal materials. There is possible observe and compare signal loss with a signal strength travelling through an environment without any obstacles (Figure 13), (Table 2). The maximum distance for control switching actuator using USB interface and SmartHomeApp visualization is 96 meters. 


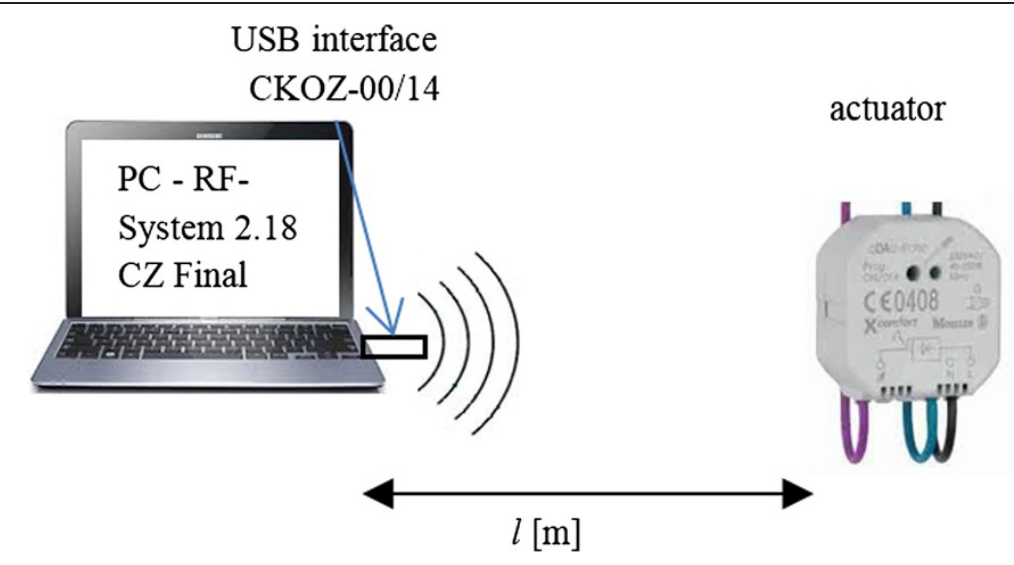

Figure 9 Block diagram of distance /[m] measurement in environment without obstacle.

During these measurements was established the optimal distance $20 \mathrm{~m}$ for switch actuator and dimming actuator and $10 \mathrm{~m}$ for shutter actuator (Table 2). The parameterization interface experiences serious problems trying to find active components in distances over 20 meters. This also limits the measurement of the signal quality.

\section{The distance /[m] measurement -environment with two brick walls}

We have also simulated real-life conditions using an environment with two brick walls. This represents a situation when the signal needs to travel through one room for example, through a corridor between two rooms (Figure 11).

It is clear that in this scenario the signal strength travelling through two brick walls rapidly deteriorates (Figure 14), (Table 3).

In our simulated environment the first wall was placed directly behind the USB communication interface and the second wall was placed two meters from the first one. During these measurements was established the optimal distance $20 \mathrm{~m}$ for switch actuator and dimming actuator and $10 \mathrm{~m}$ for shutter actuator (Table 3). The parameterization interface experiences serious problems trying to find active components in distances over 20 meters. This also limits the measurement of the signal quality.

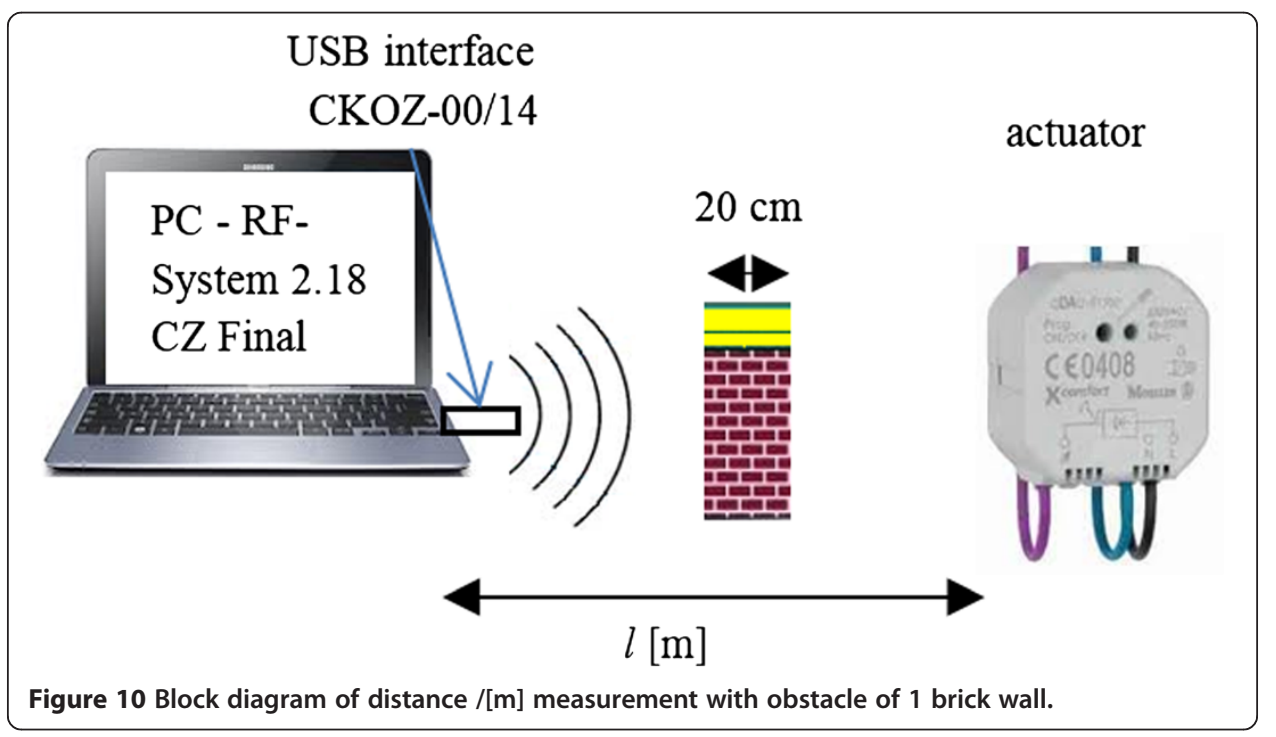




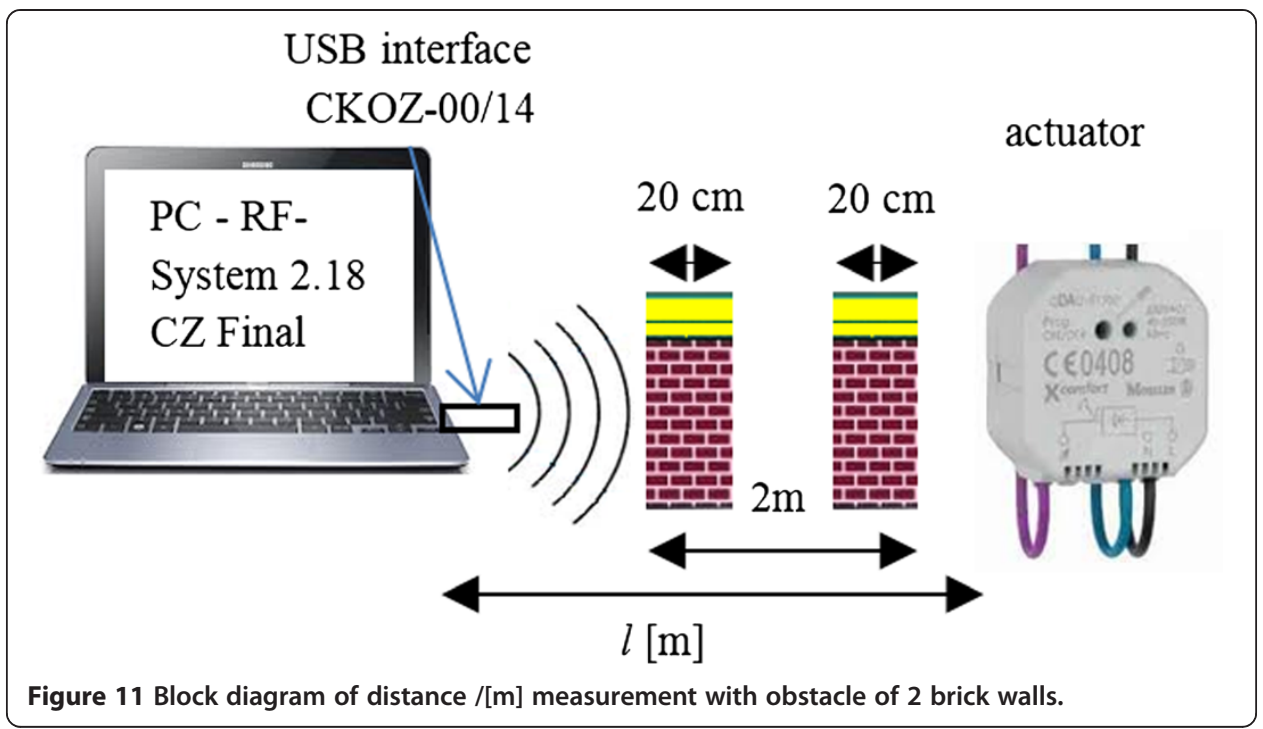

\section{Discussion}

The future development of visualization and intelligent houses.The rapid development of technologies available for houses today has created a situation where several electronic systems operate simultaneously in one house. Therefore, the integration of various installed technologies has become an important focus of many manufacturers producing these systems [22]. An automation control system which uses a wireless radio frequency may be integrated into these systems thanks to visualization tools. So this would enable us to have all technologies in one place and only distribute them around the house. One way to achieve this situation is to create a home multimedia center, where the functions of a multimedia server are handled by a small, low -power computer. Connecting to the Internet via a large number of computer peripherals allows us to connect many technologies that otherwise operate independently. As an example we can use integration of television, radio, and control of electrical systems and the Internet. Thanks to Wi-Fi [23], other devices at home may access the Internet as well. At the same time we can use a multimedia server to listen to Internet radios, or watch TV and store movies on a multimedia server, access the Internet and play computer games. Thanks to visualization,

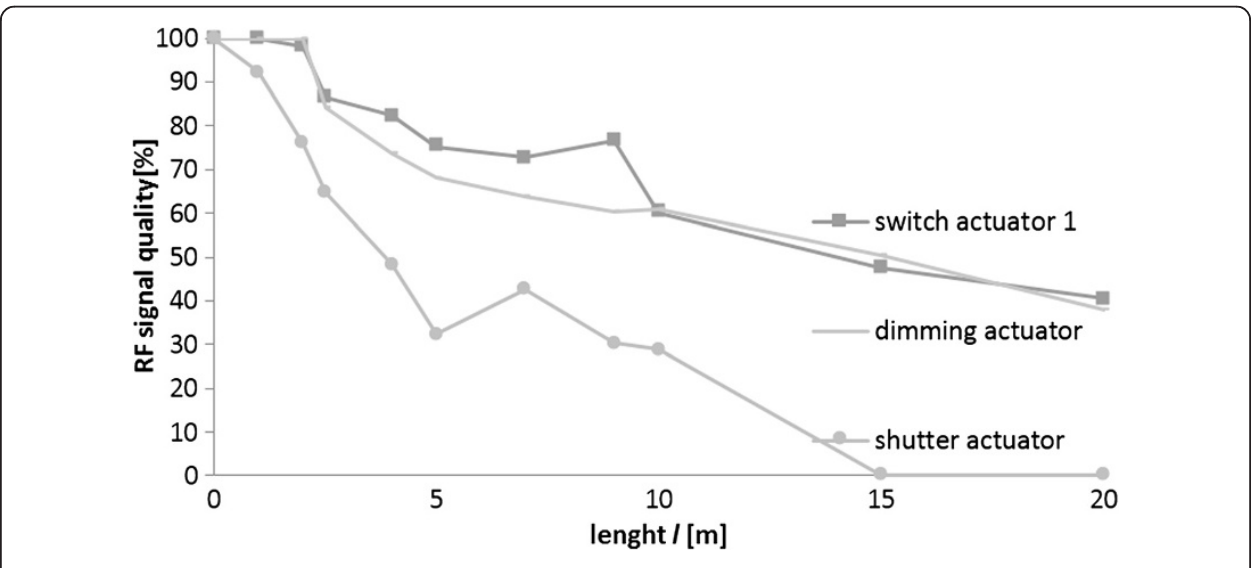

Figure 12 Graph with measured values of wireless signal quality in the environment without obstacle. 
Table 1 The distance measurement in environment without any obstacle

\begin{tabular}{|c|c|c|c|c|c|}
\hline \multicolumn{2}{|l|}{ Switch actuator } & \multicolumn{2}{|l|}{ Shutter actuator } & \multicolumn{2}{|c|}{ Dimming actuator } \\
\hline $\begin{array}{l}\text { Distance (from } \\
\text { the source) }\end{array}$ & $\begin{array}{l}\text { RF signal } \\
\text { quality }\end{array}$ & $\begin{array}{l}\text { Distance (from } \\
\text { the source) }\end{array}$ & RF signal quality & $\begin{array}{l}\text { Distance (from } \\
\text { the source) }\end{array}$ & $\begin{array}{l}\text { RF signal } \\
\text { quality }\end{array}$ \\
\hline$[[\mathrm{m}]$ & [\%] & $/[\mathrm{m}]$ & [\%] & $/[\mathrm{m}]$ & [\%] \\
\hline 0 & 100 & 0 & 100 & 0 & 100 \\
\hline 1 & 100 & 1 & 92.2 & 1 & 100 \\
\hline 2 & 98.1 & 2 & 76 & 2 & 100 \\
\hline 2.5 & 86.6 & 2.5 & 64.7 & 2.5 & 84.2 \\
\hline 4 & 82.3 & 4 & 48.3 & 4 & 73.6 \\
\hline 5 & 75.3 & 5 & 32.4 & 5 & 68.2 \\
\hline 7 & 72.8 & 7 & 42.6 & 7 & 63.7 \\
\hline 9 & 76.5 & 9 & 30.3 & 9 & 60.3 \\
\hline 10 & 60.1 & 10 & 28.8 & 10 & 60.8 \\
\hline 15 & 47.6 & 15 & 0 & 15 & 50.4 \\
\hline 20 & 40.5 & 20 & 0 & 20 & 38 \\
\hline
\end{tabular}

electrical wiring may also be easily controlled because the multimedia server can distribute the visualization system to other devices, such as smartphones, for example.

\section{Integration of visualization}

The designed visualization program is a software tool used to control the operational and technical functions of the relevant electrical system. Based on the process described in the analysis, this tool can be created using various programming languages/codes. The proposed solution for the integration of visualization was created with regard to ASP. NET technology and the $C$ \# language. The aim here is not the definition of an actual solution, but to demonstrate an alternative where this technology may take your house.

\section{Raspberry Pi}

Raspberry Pi is a computer the size of a credit card; it is small and energy-efficient, yet fully usable as a regular PC. Thanks to its small size and very low energy consumption, it is the

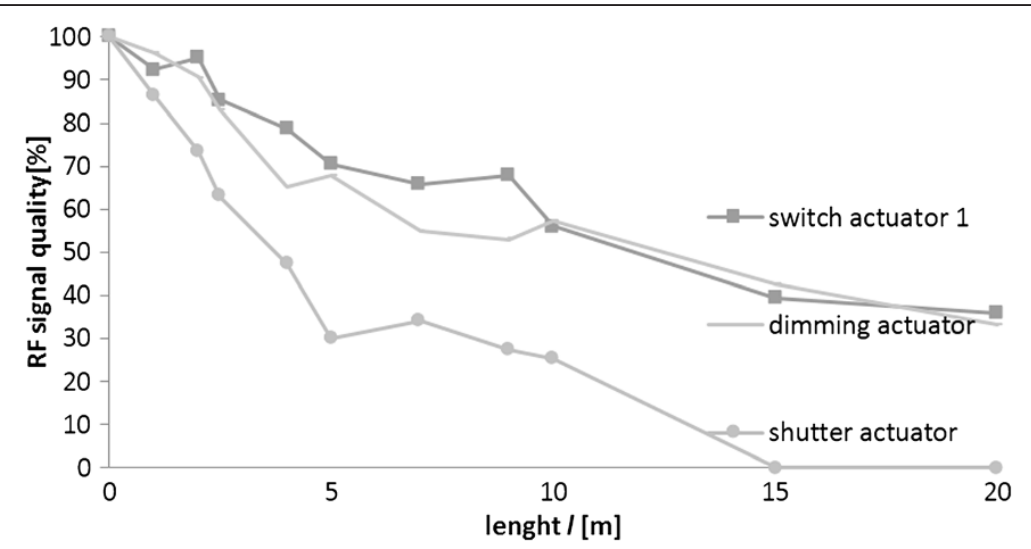

Figure 13 Graph with measured values of wireless signal quality in environment with obstacle of 1 brick wall. 
Table 2 The distance measurement in environment with one obstacle

\begin{tabular}{|c|c|c|c|c|c|}
\hline \multicolumn{2}{|l|}{ Switch actuator } & \multicolumn{2}{|l|}{ Shutter actuator } & \multicolumn{2}{|c|}{ Dimming actuator } \\
\hline $\begin{array}{l}\text { Distance (from } \\
\text { the source) }\end{array}$ & $\begin{array}{l}\text { RF signal } \\
\text { quality }\end{array}$ & $\begin{array}{l}\text { Distance (from } \\
\text { the source) }\end{array}$ & $\begin{array}{l}\text { RF signal } \\
\text { quality }\end{array}$ & $\begin{array}{l}\text { Distance (from } \\
\text { the source) }\end{array}$ & $\begin{array}{l}\text { RF signal } \\
\text { quality }\end{array}$ \\
\hline$/[\mathrm{m}]$ & [\%] & $I[\mathrm{~m}]$ & [\%] & $I[\mathrm{~m}]$ & [\%] \\
\hline 0 & 100 & 0 & 100 & 0 & 100 \\
\hline 1 & 92.4 & 1 & 86.2 & 1 & 96.4 \\
\hline 2 & 95.1 & 2 & 73.5 & 2 & 90.6 \\
\hline 2.5 & 85.3 & 2.5 & 63 & 2.5 & 83.1 \\
\hline 4 & 78.8 & 4 & 47.2 & 4 & 65.3 \\
\hline 5 & 70.6 & 5 & 29.9 & 5 & 67.8 \\
\hline 7 & 65.8 & 7 & 34 & 7 & 54.8 \\
\hline 9 & 67.7 & 9 & 27.3 & 9 & 53 \\
\hline 10 & 56 & 10 & 25.2 & 10 & 57.3 \\
\hline 15 & 39.5 & 15 & 0 & 15 & 42.6 \\
\hline 20 & 35.9 & 20 & 0 & 20 & 33.1 \\
\hline
\end{tabular}

ideal solution for continuously running applications. This makes it possible to use this device as a multimedia server, and in case of visualization also as a web server. The manufacturer offers an ARM operating system distributed as Linux versions Debian and Arch.

\section{Database}

A designed visualization program is a software product that utilizes many advanced features and large database machines. It is not necessary to use robust database servers such as MS SQL or Oracle. Compact and small databases may offer many benefits, in particular during distribution.

\section{SQLite}

The difference between a database built on a client server and SQLite is that the database server is not running as an independent process. SQLite is a small library that can be used simply by adding links or by adding a simple interface. Each SQLite database is stored in a .dbm file. The entire library is developed in $\mathrm{C}$ language and requires very low resources.

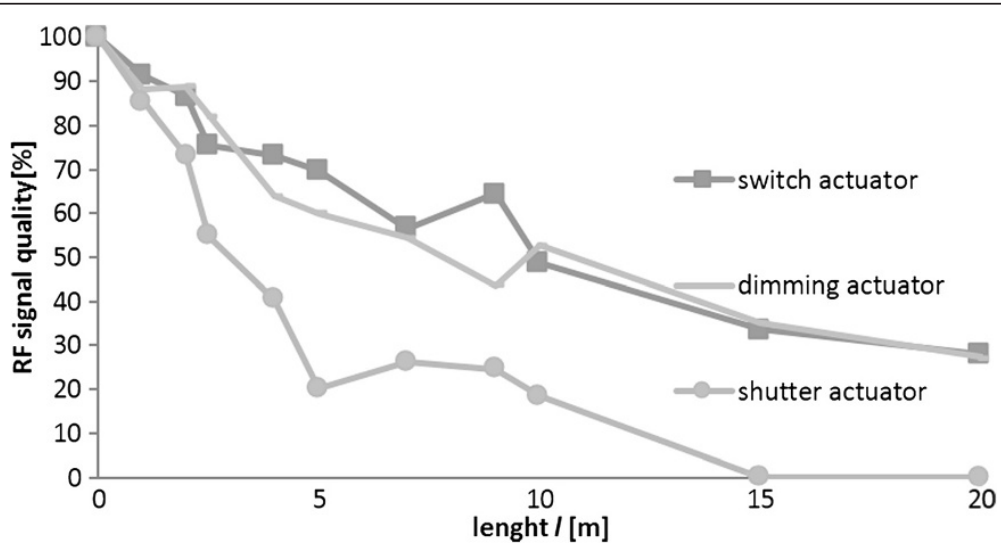

Figure 14 Graph with measured values of wireless signal quality in environment with obstacle of 2 brick walls. 
Table 3 The distance measurement in environment with two obstacles

\begin{tabular}{|c|c|c|c|c|c|}
\hline \multicolumn{2}{|l|}{ Switch actuator } & \multicolumn{2}{|l|}{ Shutter actuator } & \multicolumn{2}{|c|}{ Dimming actuator } \\
\hline $\begin{array}{l}\text { Distance (from } \\
\text { the source) }\end{array}$ & $\begin{array}{l}\text { RF signal } \\
\text { quality }\end{array}$ & $\begin{array}{l}\text { Distance (from } \\
\text { the source) }\end{array}$ & $\begin{array}{l}\text { RF signal } \\
\text { quality }\end{array}$ & $\begin{array}{l}\text { Distance (from } \\
\text { the source) }\end{array}$ & $\begin{array}{l}\text { RF signal } \\
\text { quality }\end{array}$ \\
\hline$/[\mathrm{m}]$ & [\%] & $I[\mathrm{~m}]$ & [\%] & $/[\mathrm{m}]$ & [\%] \\
\hline 0 & 100 & 0 & 100 & 0 & 100 \\
\hline 1 & 91.4 & 1 & 85.5 & 1 & 88.3 \\
\hline 2 & 86.6 & 2 & 73.2 & 2 & 88.8 \\
\hline 2.5 & 75.5 & 2.5 & 54.8 & 2.5 & 82.5 \\
\hline 4 & 73 & 4 & 40.4 & 4 & 64 \\
\hline 5 & 69.7 & 5 & 20.1 & 5 & 60 \\
\hline 7 & 56.5 & 7 & 26.2 & 7 & 54.4 \\
\hline 9 & 64.5 & 9 & 24.6 & 9 & 43.6 \\
\hline 10 & 48.7 & 10 & 18.5 & 10 & 52.7 \\
\hline 15 & 33.6 & 15 & 0 & 15 & 35.1 \\
\hline 20 & 28 & 20 & 0 & 20 & 27.3 \\
\hline
\end{tabular}

The library itself uses only 25 kilobytes of memory. Thanks to these characteristics, it is the ideal candidate for microcomputers such as Raspberry Pi.

\section{ASP.NET and C\#}

ASP.NET and C\# are Microsoft technologies. However, if we are talking about Raspberry Pi and Linux distributions, such as the Debian system, there is no need to disregard these solutions.

\section{Mono project}

This is an open-source implementation of the NET Framework, according to official ECMA standards. With the real binary compatibility it is possible to put these files directly in Mono, even if they were created in the NET Framework.

\section{Assessment of smart house development progress}

The potential of the solution presented here is great. Thanks to the versatility of Raspberry Pi, really deep integration of electronic devices may be achieved. This is one of the ways visualization may eventually go and reach people interested in smart systems. It is a path that can bring a lot of benefits in terms of distributions, as it can deliver more variable solutions, and simplify implementation from the customers perspective, or from the point of view of the technician who is installing the system.

\section{Conclusions}

The first part of the article describes the development of the visualization application software SmartHomeApp and the structure with a wireless system xComfort for comfortable control of a building service system in the Smart Home or in the Smart Home Care. The visualization application program described above is designed as a web application using DBMS MS SQL to save operational data. In terms of communication between 
the database and the visualization and active elements, a software driver was designed, which makes this communication possible.

Visualization was designed with regard to web interface requirements, ability to control the software via a mobile phone and also with regard to easy expandability, scalability and modularity. Using modern technologies ASP.NET, MS SQL, NET C\# allowed us to meet all these requirements. Availability of optimized web visualization application for various de-vices was achieved thanks to the use of HTML 5 and CSS 3 technologies. In addition to independent saving process of requirements affecting active system element behavior, data may also be used for presentation demonstrating the history of the system use. Data may also be presented in a certain way as to allow later optimization of the entire intelligent electrical installation. Further, an option to integrate web cameras and voice control into the system were also examined. The MyVoice software was used to control the visualization by voice and visualization requirements for integration between those two elements were also described. The second part of the article describes the testing method of the reliability of wireless control system for technical and operational functions control. Measured values correspond with tolerances shown in the USB communication device technical documentation. Even though the signal quality was rather low, there is possible active components control reliably and without any information loss. The quality of RF signal was measured with using parameterization interface. Measuring was done in a real apartment and not in a laboratory. Measuring of maximum communication distances between the actuator (switching, dimming, window blinds) and USB communication interface was done in all measured environments including. During these measurements was established the optimal distance $10 \mathrm{~m}$ for of the all wireless elements control.

Competing interests

The authors declare that they have no competing interests.

\section{Authors contributions}

All authors contributed to the content of this paper. All authors read and approved the final manuscript.

\section{Acknowledgements}

This paper has been elaborated in the framework of the project Opportunity for young researchers, reg. no. CZ.1.07/ 2.3.00/30.0016, supported by Operational Program Education for Competitiveness and co-financed by the European Social Fond and the state budget of the Czech Republic. This work was supported by project SP2014/156,

Microprocessor based systems for control and measurement applications. of Student Grant System, VSB-TU Ostrava.

Received: 3 October 2014 Accepted: 20 November 2014

Published online: 31 December 2014

\section{References}

1. Vanus J, Koziorek J, Hercik R (2013) Design of a smart building control with view to the senior Citizens needs. Programmable Devices and Embedded Systems 12(1):422 427

2. Amirabdollahian F, Op den Akker R, Bedaf S, Bormann R, Draper H, Evers V, Gelderblom GJ, Ruiz CG, Hewson D, Hu N, lacono I, Koay KL, Krose B, Marti P, Michel H, Prevot-Huille H, Reiser U, Saunders J, Sorell T, Dautenhahn K (2013) Accompany: Acceptable robotiCs COMPanions for AgeiNg Years - Multidimensional Aspects of HumanSystem Interactions, Human System Interaction (HSI). The 6th International Conference, Sopot, pp 570 577, Doi: 10.1109/HSI.2013.6577882

3. Basu D, Moretti G, Sen Gupta G, Marsland S (2013) Wireless Sensor Network Based Smart Home: Sensor Selection, Deployment and Monitoring, Sensors Applications Symposium (SAS). IEEE, Galveston, TX, pp 49 54, Doi: 10.1109/ SAS.2013.6493555

4. Beaudin JS, Intille SS, Morris ME (2006) To track or not to track: user reactions to concepts in longitudinal health monitoring. J Med Internet Res 8(4):e29, http://www.jmir.org/2006/4/e29, Doi: 10.2196/jmir.8.4.e29

5. Booysen MJ, Gilmore JS, Zeadally S, Van Rooyen GJ (2012) Machine-to-Machine (M2M) Communications in Vehicular Networks. Ksii Transactions on Internet and Information Systems 6(2):529 546

6. Population prognosis Czech republic http://www.czso.cz/csu/2004edicniplan.nsf/p/4025-04 [cit. 2014-3-11]

7. Catala A, Pons P, Jaen J, Mocholi JA, Navarro E (2013) A meta-model for dataflow-based rules in smart environments: evaluating user comprehension and performance. Sci Comput Program 78(10):19301950

8. Choi A, Woo W (2010) Daily physiological signal monitoring system for fostering social well-being in smart spaces Cybern Syst 41(3):262 279 
9. Fercher AJ, Hitz M, Leitner G (2009) Raising awareness of energy consumption in smart living environments. In: Callaghan V, Kameas A, Reyes A, Royo D, Weber M (eds) Conference: Intelligent Environments 2009 - Proceedings of the 5th International Conference on Intelligent Environments. Intelligent Environments, Barcelona, Spain, pp 91 98, Doi: 10.3233/978-1-60750-034-6-91

10. Ghidini G, Das SK, Gupta V, leee (2012) FuseViz: A Framework for Web-Based Data Fusion and Visualization in Smart Environments. 9th leee International Conference on Mobile Ad-Hoc and Sensor Systems, pp 468472

11. Giacomin J, Bertola D (2012) Human emotional response to energy visualisations. Int J Ind Ergon 42(6):542 552

12. Fleck S, Loy R, Vollrath C, Walter F, Strasser W (2007) Smartclassysurv - A Smart Camera Network for Distributed Tracking and Activity Recognition and Its Application to Assisted Living, ICDSC '07. First ACM/IEEE International Conference, 25-28 Sept. 2007. Distributed Smart Cameras, Vienna, p 211, Doi: 10.1109/ICDSC.2007.4357526

13. Lee JY, Seo D, Rhee GW, Hong SH, Nam JS (2008) Virtual and Pervasive Smart Home Services Using Tangible Mixed Reality, Parallel and Distributed Processing with Applications, 2008. ISPA '08. International Symposium, Sydney, NSW, pp 403 408, Doi: 10.1109/ISPA.2008.92

14. Pecka A, Osadcuks V (2010) Discrete Indicators and Touch-Panel Based Human-Machine Interface. Applied Information and Communication Technologies, Jelgava, Latvia, pp 295300

15. Su J-M, Huang C-F (2014) An easy-to-use 3D visualization system for planning context-aware applications in smart buildings. Computer Standards \& Interfaces 36(2):312 326

16. Su ZL, Wang RM, Wang Z, Xie HJ (2010) Channel Service for the Digital Home Oriented Textile Consumption. 3rd International Symposium of Textile Bioengineering and Informatics, Shanghai, Peoples R China, pp 154 158, Doi: 10.3993/tbis2010029

17. Zheng $\mathrm{H}$, Wang $\mathrm{H}$, Black N, leee (2008) Human activity detection in smart home environment with self-adaptive neural networks. Proceedings of 2008 leee International Conference on Networking, Sensing and Control 1 and 2:1505 1510

18. Vanus J, Kucera P, Koziorek J (2014) The software analysis used for visualization of technical functions control in smart home care, 3rd computer science on-line conference 2014 (CSOC 2014). Series: Advances in Intelligent Systems and Computing 285:549 558, doi:10.1007/978-3-319-06740-7_47

19. Vanus J, Kucera P, Koziorek J (2014) Visualization software designed to control operational and technical functions in smart homes, 3rd computer science on-line conference 2014 (CSOC 2014). Series: Advances in Intelligent Systems and Computing 285:559 569, doi:10.1007/978-3-319-06740-7_48

20. Martinek R, Al-Wohaishi M, Zidek J (2010) Software Based Flexible Measuring Systems for Analysis of Digitally Modulated Systems. In Roedunet International Conference (RoEduNet), 2010, 9th edn. IEEE, , pp 397402

21. Chorianopoulos K (2013) Collective intelligence within web video. Human-centric Computing and Information Sciences 3:10, doi:10.1186/2192-1962-3-10

22. Augusto JC, Callaghan V, Cook D, Kameas A, Satoh I (2013) Intelligent Environments: a manifesto. Human-centric Computing and Information Sciences 3:12, doi:10.1186/2192-1962-3-12

23. Luo Y, Hoeber O, Chen $Y$ (2013) Enhancing Wi-Fi fingerprinting for indoor positioning using human-centric collaborative feedback. Human-centric Computing and Information Sciences 3:2, doi:10.1186/2192-1962-3-2

\section{Submit your manuscript to a SpringerOpen ${ }^{\circ}$} journal and bene区t from:

- Convenient online submission

- Rigorous peer review

- Immediate publication on acceptance

- Open access: articles freely available online

- High visibility within the 囚eld

- Retaining the copyright to your article 